\title{
Cerebellar Scholars' Challenging Time in COVID-19 Pandemia
}

\author{
Aasef G. Shaikh ${ }^{1} \cdot$ Hiroshi Mitoma ${ }^{2} \cdot$ Mario Manto $^{3,4}$ \\ Published online: 16 April 2020 \\ (C) Springer Science+Business Media, LLC, part of Springer Nature 2020
}

\begin{abstract}
Novel coronavirus, SARS-CoV2, has caused pandemic of highly contagious disease called coronavirus disease 2019 (COVID19), with epicenters in China, Italy, Spain, and the USA. Primarily affecting the human respiratory system, SARS-CoV2 has some impact on the human brain, but apparently minimal on the cerebellum, at least so far. Neurological involvement in the acute phase appears to manifest with confusion, dizziness, impaired consciousness, propensity to develop acute strokes, anosmia, hypogeusia, ataxia, epilepsy, and neuralgia. Cerebellar scholars are facing a time of uncertainty. Telemedicine has suddenly emerged as an alternative to follow patients. There is an urgent need to develop novel platforms to assess and follow ataxic patients remotely, especially because cerebellar patients often require ambulatory care to maintain their autonomy.
\end{abstract}

This editorial will serve as a note of greetings and to wish the cerebellum community the best of their health and productivity in theoretical research, writing and analyzing existing experimental dataset, as well as preparing the future for the next generation of students. We are going through the challenging times of a deadly pandemic, deprived socially to meet colleagues and friends, homebound to focus on priorities of daily life. The source of the current global challenge is the malignantly pathogenic coronavirus that emerged from Wuhan, China, leading to highly contagious and novel condition called coronavirus disease 2019 (COVID-19).

The core manifestation of COVID-19 includes fever in up to $98 \%[1,2]$, cough in up to $82 \%[1,2]$, shortness of breath in $31 \%$ [2], myalgia or fatigue in up to $44 \%$ [1], and headache in $8 \%[1,2]$. Fast tracked literature revealed that in its acute phase, compared with the impact on the respiratory system, the human brain appears relatively spared, generally causing confusion (9\%), dizziness (17\%), impaired consciousness $(8 \%)$, propensity to develop acute strokes (3\%), anosmia

Mario Manto

mmanto@ulb.ac.be

1 Louis Stokes Cleveland VA Medical Center, University Hospitals Cleveland Medical Center, Cleveland, $\mathrm{OH}$, USA

2 Department of Medical Education, Tokyo Medical University, Tokyo, Japan

3 Unité des Ataxies Cérébelleuses, Service de Neurologie, CHU-Charleroi, 6000 Charleroi, Belgium

4 Service des Neurosciences, University of Mons, 7000 Mons, Belgium
(6\%), hypogeusia (6\%), acute cerebrovascular diseases (3\%), ataxia (1\%), epilepsy (1\%), and neuralgia [3]. Details on so-called ataxic signs are missing. While reported neurological deficits were from those who were symptomatic, the "asymptomatic" patients had commonly experienced anosmia and hypogeusia [4]. Respiratory distress is one of the recognized causes of the morbidity and mortality of this disease obviously, and impairment in central respiratory drive secondary to the brainstem involvement is plausible [5, 6]. Our colleagues in Italy have treated increasing numbers of patients with cerebrovascular events; the virus might affect coagulation mechanisms. While in acute phase COVID-19 does not seem to involve the cerebellum, we do not know its chronic consequences. We are especially worried about the consequences of social isolation on the cerebellar patients who are dependent on rehabilitation services and exhibit emotional, social, or cognitive fragility.

Experts sadly believe that some patients who are affected with COVID-19 will not live longer enough to see its chronic consequence. As of now, COVID-19 has affected millions people worldwide and has taken hundreds of thousands of lives, and the global census of COVID-19-positive patients is growing day-by-day. As much as the reality associated with this malignant fraction of RNA, the fear of contracting it makes our world anxious. Often, people do not know what to believe and what not to believe. Call it a response to anxiety, defense mechanism, or simply a sense that "we are all in the same boat"; we have been noticing a sense of warmth and care around us, not only from the near and dear ones but also from total strangers. We feel that people have become less selfish, more cognizant, and understanding. 
Several questions arise: how to maximize our ability, how to utilize remote monitoring practices, and satellite meetings. Social isolation, shortage of equipment, and difficulties for face-to-face teaching and mentoring are pushing telemedicine. We have minimal doubt that such a practice pattern if followed even after we get relief from COVID-19 will only do better for our professional and personal life. The cerebellum community will not be an exception to this novel rule.

We believe that the coronavirus pandemic has been an eyeopener, teaching important life lessons:

1. We learnt that most $(80 \%$ ?) of the academic or administrative work we do can be done in isolation (without a big group). Among the remaining 20\%, $80 \%$ can be done by remote consulting. This experience should make everyone to be self-sufficient, we need to spend less time in organizing in person meeting, spending less time (and fuel) traveling from point A to B, and spend more time in doing actual work. Altogether, it will only do better for the world's development and environment. This also applies to the cerebellum community.

2. Money is abundant if used wisely. Most of the times, more than $60 \%$ of our need can be satisfied with less than $40 \%$ of the money we have in grant funding.

3. Every human is a super-human. We can develop infrastructures very rapidly. We can create hospitals, start and complete clinical trials, develop medicines, convert distilleries and wineries to produce hand sanitizers, and transform vacuum cleaner production plant to make ventilators within a matter of days. Students on their side are producing masks and equipment protection for free.

4. Family and professional life do not reciprocate. We can spend time at home with the family under hygienic guidelines; we still can get the work done and professionally do well.

5. We can survive and have quality time with the family without having expensive vacation at luxurious resorts.

6. Developed nations and wealthy people are as vulnerable as poor.

Ataxiologists along with their fellow scientists are encountering difficulties to accomplish research in labs and to gather funding. Ongoing works of $\mathrm{PhD}$ students have been suddenly blocked. Nevertheless, we are confident that our scholarly cerebellar colleagues are still at their best establishing a strong example community during these trying times. We have very little doubt that in the near future, there will be a surge of scholarly publication in the cerebellar field, especially theoretical work and analysis of previously collected datasets. Such academic productivity will be the result of optimized time; it will do nothing but excellent to fight the chronic neurodegenerative condition that is as bad as COVID-19 and is affecting the humankind at a slow pace.

On the clinical side, the telemedicine has emerged as an excellent alternative to follow patients and keep them out of isolation. Unfortunately, ambulatory services are often closed and rehabilitation services at home and in rest houses or assisted living facilities have dropped dramatically, impacting in particular on deambulation. Restrictions are impacting on autonomy of patients. There is an urgent need to develop novel platforms to assess and follow ataxic patients remotely. This needs to include not only the sensorimotor aspects but also neuropsychological care and therapies administered. A typical example is the difficulties to perform studies requiring close contacts with patients. Cerebellar patients are particularly vulnerable. While the cerebellar scholars prepare the scientific infrastructure, we urge the healthcare authorities to help us promote these novel platforms. The cerebellum community has a major role to play to validate these novel technologies at a worldwide level. There was a time before COVID-19 and there will be a time after COVID-19.

\section{Compliance with Ethical Standards}

Conflict of Interest The authors declare that they have no conflict of interest.

\section{References}

1. Huang C, Wang Y, Li X, Ren L, Zhao J, Hu Y, et al. Clinical features of patients infected with 2019 novel coronavirus in Wuhan, China. Lancet. 2020;395:497-506.

2. Chen N, Zhou M, Dong X, Qu J, Gong F, Han Y, et al. Epidemiological and clinical characteristics of 99 cases of 2019 novel coronavirus pneumonia in Wuhan, China: a descriptive study. Lancet. 2020;395:507-13.

3. Mao L, Wang M, Chen S, He Q, Chang J, Hong CD, et al. Neurological manifestations of hospitalized patients with COVID19 in Wuhan, China: a retrospective case series study. MedRxiv. https://doi.org/10.1101/2020.02.22.20026500.

4. Giacomelli A, Pezzati L, Conti F, Bernacchia D, Siano M, Oreni L, et al. Self-reported olfactory and taste disorders in SARS-CoV-2 patients: a cross sectional study. Clin Infect Dis. 2020 Mar 26. pii: ciaa330. doi: https://doi.org/10.1093/cid/ciaa330.

5. Li YC, Bai WZ, Hashikawa T. The neuroinvasive potential of SARSCoV2 may play a role in the respiratory failure of COVID-19 patients. J Med Virol. 2020. https://doi.org/10.1002/jmv.25728.

6. Baig AM, Khaleeq A, Ali U, Syeda H. Evidence of the COVID-19 virus targeting the CNS: tissue distribution, host-virus infection, and proposed neurotropic mechanisms. ACS Chem Neurosci. 2020 Mar 13. https://doi.org/10.1021/acschemneuro.0c00122.

Publisher's Note Springer Nature remains neutral with regard to jurisdictional claims in published maps and institutional affiliations. 